\title{
Risk Factors and Antipsychotic Usage Patterns Associated With Terminal Delirium in a Veteran Long-Term Care Hospice Population
}

\author{
Emily M. Ellsworth, PharmD, BCGP; Kevin J. Bacigalupo, PharmD, BCPS, BCGP; Kavita R. Palla, PharmD, BCPS; \\ Seema S. Limaye, MD; Margaret J. Walkosz, ACHPN, GNP-BC; Sandra T. Szczecinski, RN, BSN; \\ and Katie J. Suda, PharmD, MS
}

Background: The development of delirium is very common
in terminally ill patients. However, risk factors for termi-
nal delirium in the veteran population are poorly identified.
The purpose of this study was to (1) Identify risk factors for
terminal delirium in a US Department of Veterans Affairs
inpatient hospice population; (2) Assess usage patterns
of antipsychotics for treatment of terminal delirium; and
(3) Describe nursing assessment, nonpharmacologic inter-
ventions, and documentation of terminal delirium. Methods: This was a retrospective case-control study of veterans who expired while admitted into hospice care at a long-term care hospice unit during the period of October 1, 2013 to September 30, 2015. Veterans' medical records were reviewed for the 2 weeks prior to the recorded death.
Results: Of 307 veterans admitted for hospice care, $67.4 \%$ required antipsychotics in the last 2 weeks of life for the treatment of terminal delirium. The average number of antipsychotic doses given was 14.9 doses per patient. The risk factors that were identified included the use of steroids, opioids, or anticholinergics; Vietnam-era veterans with liver disease; veterans with cancer and a comorbid mental health disorder; and veterans with a history of drug and/or alcohol abuse.

Conclusions: More than half of veterans admitted for hospice care experienced terminal delirium requiring treatment with antipsychotics. The identification of veterans most likely to develop terminal delirium will allow for early nonpharmacologic interventions and potentially decrease the need for treatment with antipsychotic medications.

\section{Author affiliations} can be found at the end of the article. Correspondence: Emily Ellsworth (emily.ellsworth2@va.gov)

Fed Pract. 2021;38(5). Published online May 12. doi:10.12788/fp.0131
D elirium is a condition commonly exhibited by hospitalized patients and by those who are approaching the end of life. ${ }^{1}$ Patients who experience a disturbance in attention that develops over a relatively short period and represents an acute change may have delirium. ${ }^{2}$ Furthermore, there is often an additional cognitive disturbance, such as disorientation, memory deficit, language deficits, visuospatial deficit, or perception. Terminal delirium is defined as delirium that occurs in the dying process and implies that reversal is less likely. ${ }^{3}$ When death is anticipated, diagnostic workups are not recommended, and treatment of the physiologic abnormalities that contribute to delirium is generally ineffective. ${ }^{4}$

\section{BACKGROUND}

Delirium is often underdiagnosed and undetected by the clinician. Some studies have shown that delirium is not detected in 22 to $50 \%$ of cases. ${ }^{5}$ Factors that contribute to the underdetection of delirium include preexisting dementia, older age, presence of visual or hearing impairment, and hypoactive presentation of delirium. Other possible reasons for nondetection of delirium are its fluctuating nature and lack of formal cognitive assessment as part of a routine screening across care settings. ${ }^{5}$ Another study found that $41 \%$ of health care providers (HCPs) felt that screening for delirium was burdensome. ${ }^{6}$

To date, there are no veteran-focused studies that investigate prevalence or risk factors for terminal delirium in US Department of Veterans Affairs (VA) longterm care hospice units. Most long-term care hospice units in the VA are in community living centers (CLCs) that follow regulatory guidelines for using antipsychotic medications. The Centers for Medicare and Medicaid Services state that if antipsychotics are prescribed, documentation must clearly show the indication for the antipsychotic medication, the multiple attempts to implement planned care, nonpharmacologic approaches, and ongoing evaluation of the effectiveness of these interventions. ${ }^{7}$ The symptoms of terminal delirium cause significant distress to patients, family and caregivers, and nursing staff. Literature suggests that delirium poses significant relational challenges for patients, families, and HCPs in end-oflife situations. ${ }^{89}$ We hypothesize that the early identification of risk factors for the development of terminal delirium in this population may lead to increased use of nonpharmacologic measures to prevent 
terminal delirium, increase nursing vigilance for development of symptoms, and reduce symptom burden should terminal delirium develop.

Prevalence of delirium in the long-term care setting has ranged between 1.4 and $70.3 \% .^{10}$ The rate was found to be much higher in institutionalized populations compared with that of patients classified as athome. In a study of the prevalence, severity, and natural history of neuropsychiatric syndromes in terminally ill veterans enrolled in community hospice, delirium was found to be present in only $4.1 \%$ on the initial visit and $42.5 \%$ during last visit. Also, more than half had at least 1 episode of delirium during the 90-day study period. ${ }^{11}$ In a study of the prevalence of delirium in terminal cancer patients admitted to hospice, $80 \%$ experienced delirium in their final days. ${ }^{12}$

Risk factors for the development of delirium that have been identified in actively dying patients include bowel or bladder obstruction, fluid and electrolyte imbalances, suboptimal pain management, medication adverse effects and toxicity (eg, benzodiazepines, opioids, anticholinergics, and steroids), the addition of $\geq 3$ medications, infection, hepatic and renal failure, poor glycemic control, hypoxia, and hematologic disturbances. ${ }^{4,5,13} \mathrm{~A}$ high percentage of patients with a previous diagnosis of dementia were found to exhibit terminal delirium. ${ }^{14}$

There are 2 major subtypes of delirium: hyperactive and hypoactive. ${ }^{4}$ Patients with hypoactive delirium exhibit lethargy, reduced motor activity, lack of interest, and/ or incoherent speech. There is currently little evidence to guide the treatment of hypoactive delirium. By contrast, hyperactive delirium is associated with hallucinations, agitation, heightened arousal, and inappropriate behavior. Many studies suggest both nonpharmacologic and pharmacologic treatment modalities for the treatment of hyperactive delirium. ${ }^{4,13}$ Nonpharmacologic interventions may minimize the risk and severity of symptoms associated with delirium. Current guidelines recommend these interventions before pharmacologic treatment. ${ }^{4}$ Nonpharmacologic interventions include but are not limited to the following: engaging the patient in mentally stimulating activities; surrounding the patient

\section{Case Presentation}

RB was a 69-year-old veteran who enlisted in the US Army after high school and served in a combat zone in Vietnam. On returning home, he experienced mood swings and nightmares and was diagnosed with posttraumatic stress disorder (PTSD). For several years he struggled with polysubstance misuse but managed to overcome his addictions to everything but tobacco. He considered his nightly consumption of 6 beers to be normal for an American male.

After a syncopal episode and fall while mowing his lawn, he was diagnosed with metastatic lung cancer that was too advanced to benefit from surgery or chemotherapy. He was prescribed dexamethasone for edema surrounding the brain lesions and admitted to a US Department of Veterans Affairs long-term care hospice unit while receiving palliative whole-brain radiation. He remained stoic and functionally independent throughout the treatment but became progressively weaker with decreasing intake of food and fluids. His pain escalated, and he required opioids around-the-clock.

A few days before his death, he became agitated, confused, and combative. He fell while trying to get to the bathroom on his own. While staff members were trying to assist him, he began to tug at his clothes and cried out, "rats, rats are eating me... get out of my way, I need to save my children!"

RB's hyperactive terminal delirium symptoms persisted in the last few days of his life. Based on the results of this study, the hospice team identified several risk factors that put RB at risk for the development of terminal delirium, including his Vietnam-era veteran status, history of PTSD, substance abuse, and cancer with liver involvement. Nursing was informed of his risk factors and consistently incorporated nonpharmacologic methods, such as adjusting lighting in the room, monitoring his bowel and bladder needs, and treating his pain. A trial dose of lorazepam administered for anxiety was ineffective. He ultimately calmed after receiving 4 doses of haloperidol in a 24-hour period. Due to his distress and concerns for his safety, haloperidol was scheduled every 6 hours. He proceeded to a comfortable death.

with familiar materials (eg, photos); ensuring that all individuals identify themselves when they encounter a patient; minimizing the intensity of stimulation, providing family or volunteer presence, soft lighting and warm blankets; and ensuring the patient uses hearing aids and glasses if needed. ${ }^{4,14}$

Although there are no US Food and Drug Administration-approved medications to treat hyperactive delirium, firstgeneration antipsychotics (eg, haloperidol, chlorpromazine) are considered the firstline treatment for patients exhibiting psychosis and psychomotor agitation..$^{3,414-16}$ In terminally ill patients, there is limited evidence from clinical trials to support the efficacy of drug therapy. ${ }^{14}$ One study showed lack of efficacy with hydration and opioid rotation. ${ }^{17}$ In terminally ill patients experiencing hyperactive delirium, there is a significant increased risk of muscle tension, 
TABLE 1 Patient Baseline Characteristics

\begin{tabular}{|c|c|c|c|}
\hline Variables & Cases $(n=186)$ & Controls $(n=90)$ & $P$ value \\
\hline Age, mean (SD), y & $76.0(12.1)$ & $74.8(2.8)$ & .46 \\
\hline \multicolumn{4}{|l|}{ War era, No. (\%) } \\
\hline World War II & $50(26.9)$ & $21(23.3)$ & .75 \\
\hline Korea & $45(24.2)$ & $21(23.3)$ & .75 \\
\hline Vietnam & 91 (48.9) & $44(48.9)$ & .49 \\
\hline Persian Gulf & 0 & $3(3.3)$ & -- \\
\hline $\begin{array}{l}\text { Operation Enduring Freedom/ } \\
\text { Operation Iraqi Freedom }\end{array}$ & 0 & $1(1.1)$ & -- \\
\hline $\begin{array}{l}\text { Time between diagnosis and } \\
\text { death, mean (SD), mo }\end{array}$ & $16.4(24.1)$ & $25.4(39.6)$ & .22 \\
\hline \multicolumn{4}{|l|}{ Terminal diagnosis, No. (\%) } \\
\hline Cancer & $112(60.2)$ & $44(48.9)$ & .07 \\
\hline $\begin{array}{l}\text { Chronic obstructive pulmonary } \\
\text { disease }\end{array}$ & $17(9.1)$ & & \\
\hline $\begin{array}{l}\text { disease } \\
\text { Heart failure }\end{array}$ & $9(48)$ & $\begin{array}{l}8(8.9) \\
6(6.7)\end{array}$ & $\begin{array}{l}.95 \\
.53\end{array}$ \\
\hline End-stage renal disease & $6(3.2)$ & $3(3.3)$ & .09 \\
\hline Liver disease $^{\mathrm{a}}$ & $9(4.8)$ & $4(4.4)$ & .99 \\
\hline Parkinson disease & $0(0)$ & $1(1.1)$ & .33 \\
\hline Dementia & $6(3.2)$ & $6(6.7)$ & .19 \\
\hline Sepsis & $12(6.5)$ & $7(7.8)$ & .68 \\
\hline Other & $15(8.1)$ & $11(12.2)$ & .27 \\
\hline \multicolumn{4}{|l|}{ Comorbid conditions, No. (\%) } \\
\hline Cancer & $43(23.1)$ & $16(17.8)$ & .31 \\
\hline $\begin{array}{l}\text { Chronic obstructive pulmonary } \\
\text { disease }\end{array}$ & $44(23.7)$ & $21(23.3)$ & .95 \\
\hline Heart failure & $47(25.3)$ & $19(21.1)$ & .45 \\
\hline Renal disease $^{b}$ & $68(36.6)$ & $41(45.6)$ & .15 \\
\hline Liver disease $^{\mathrm{a}}$ & $37(19.9)$ & $14(15.6)$ & .38 \\
\hline Parkinson disease & $4(2.2)$ & $2(2.2)$ & .99 \\
\hline Dementia & $46(24.7)$ & 17 (18.9) & .28 \\
\hline Suspected infection & $81(43.5)$ & $51(56.7)$ & .04 \\
\hline $\begin{array}{l}\text { History of drug or alcohol } \\
\text { misuse }\end{array}$ & 62 (33.3) & $19(21.1)$ & .04 \\
\hline
\end{tabular}

${ }^{a}$ Cirrhosis, hepatitis.

${ }^{\mathrm{b}} \mathrm{Chronic}$ kidney disease, end-stage renal disease.

myoclonic seizures, and distress to the patient, family, and caregiver. ${ }^{1}$ Benzodiazepines can be considered first-line treatment for dying patients with terminal delirium in which the goals of treatment are to relieve muscle tension, ensure amnesia, reduce the risk of seizures, and decrease psychosis and agitation. ${ }^{18,19}$ Furthermore, in patients with history of alcohol misuse who are experiencing terminal delirium, benzodiazepines also may be the preferred pharmacologic treatment. ${ }^{20}$ Caution must be exercised with the use of benzodiazepines because they can also cause oversedation, increased confusion, and/or a paradoxical worsening of delirium. ${ }^{3,4,14}$

\section{METHODS}

This was a retrospective case-control study of patients who died in the Edward Hines Jr. Veterans Affairs Hospital CLC in Hines, Illinois, under the treating specialty nurs- ing home hospice from October 1, 2013 to September 30, 2015. Due to the retrospective nature of this trial, the use of antipsychotics within the last 2 weeks of life was a surrogate marker for development of terminal delirium. Cases were defined as patients who were treated with antipsychotics for terminal delirium within the last 2 weeks of their lives. Controls were defined as patients who were not treated with antipsychotics for terminal delirium within the last 2 weeks of their lives. Living hospice patients and patients who were discharged from the CLC before death were excluded.

The goals of this study were to (1) determine risk factors in the VA CLC hospice veteran population for the development of terminal delirium; (2) evaluate documentation by the nursing staff of nonpharmacologic interventions and indications for antipsychotic use in the treatment of terminal delirium; and (3) examine the current usage patterns of antipsychotics for the treatment of terminal delirium.

Veterans' medical records were reviewed from 2 weeks before death until the recorded death date. Factors that were assessed included age, war era of service, date of death, terminal diagnosis, time interval from cancer diagnosis to death, comorbid conditions, prescribed antipsychotic medications, and other medications potentially contributing to delirium. Nursing documentation was reviewed for indications for administration of antipsychotic medications and nonpharmacologic interventions used to mitigate the symptoms of terminal delirium.

Statistical analysis was conducted in SAS Version 9.3. Cases were compared with controls using univariate and multivariate statistics as appropriate. Comparisons for continuous variables (eg, age) were conducted with Student $t$ tests. Categorical variables (eg, PTSD diagnosis) were compared using $\chi^{2}$ analysis or Fisher exact test as appropriate. Variables with a $P$ value $<.1$ in the univariate analysis were included in logistic regression models. Independent variables were removed from the models, using a backward selection process. Interaction terms were tested based on significance and clinical relevance. A $P$ value $<.05$ was considered statistically significant. 


\section{RESULTS}

From October 1, 2013 to September 30, 2015, 307 patients were analyzed for inclusion in this study. Within this population, 186 received antipsychotic medications for the treatment of terminal delirium (cases), while 90 did not receive antipsychotics (controls). Of the 31 excluded patients, 13 were discharged to receive home hospice care, 11 were discharged to community nursing homes, 5 died in acute care units of Edward Hines, Jr. VA Hospital, and 2 died outside of the study period.

The mean age of all included patients was 75.5 years, and the most common terminal diagnosis was cancer, which occurred in 156 patients $(56.5 \%)$ (Table 1$)$. The baseline characteristics were similar between the cases and controls, including war era of veteran, terminal diagnosis, and comorbid conditions. The mean time between cancer diagnosis and death was not notably longer in the control group compared with that of the case group ( 25 vs 16 mo, respectively). There was no statistically significant difference in terminal diagnoses between cases and controls. Veterans in the control group spent more days (mean [SD]) in the hospice unit compared with veterans who experienced terminal delirium (48.5 [168.4] vs 28.2 [46.9]; $P=.01$ ). Patients with suspected infections were more likely found in the control group $(P=.04$; odds ratio $[O R]=1.70$; $95 \%$ CI, 1.02-2.82).

The most common antipsychotic administered in the last 14 days of life was haloperidol. In the case group, 175 (94\%) received haloperidol at least once in the last 2 weeks of life. Four (4.4\%) veterans in the control group received haloperidol for the indication of nausea/vomiting; not terminal delirium. Atypical antipsychotics were infrequently used and included risperidone, olanzapine, quetiapine, and aripiprazole.

A total of 186 veterans received at least 1 dose of an antipsychotic for terminal delirium: $97(52.2 \%)$ veterans requiring an- tipsychotics for the treatment of terminal delirium required both scheduled and asneeded doses; 75 (40.3\%) received only as-needed doses, and 14 (7.5\%) required only scheduled doses. When the number of as-needed and scheduled doses were combined, each veteran received a mean 14.9 doses. However, for those veterans with antipsychotics ordered only as needed, a mean 5.8 doses were received per patient. Administration of antipsychotic doses was split evenly among the 3 nursing shifts (day-evening-night) with about $30 \%$ of doses administered on each shift.

Nurses were expected to document nonpharmacologic interventions that preceded the administration of each antipsychotic dose. Of the 1,028 doses administered to the 186 veterans who received at least 1 dose of an antipsychotic for terminal delirium, most of the doses (99.4\%) had inadequate documentation based on current long-term care guidelines for prudent antipsychotic use. $^{9}$

Several risk factors for terminal delirium were identified in this veteran population. Veterans with a history of drug or alcohol abuse were found to be at a significantly higher risk for terminal delirium $(P=.04$; OR, 1.87; 95\% CI, 1.03-3.37). As noted in previous studies, steroid use $(P=.01$; OR, 2.57 ; 95\% CI, 1.26-5.22); opioids ( $P=.007$; OR, 5.94; 95\% CI, 1.54-22.99), 
TABLE 3 Primary Endpoint With Combined Risk Factors

\begin{tabular}{|c|c|c|c|c|}
\hline Risk Factors & $\begin{array}{l}\text { Cases, No. }(\%) \\
\quad(n=186)\end{array}$ & $\begin{array}{l}\text { Controls, No. (\%) } \\
\qquad(\mathrm{n}=90)\end{array}$ & $\begin{array}{c}P \\
\text { value }\end{array}$ & $\begin{array}{l}\text { Odds Ratio } \\
(95 \% \mathrm{Cl})\end{array}$ \\
\hline Vietnam veterans with liver disease (vs no liver disease) & $35(18.8)$ & $9(10.0)$ & .04 & $1.21(1.01-1.45)$ \\
\hline $\begin{array}{l}\text { Veterans with a history of alcohol and/or drug abuse and liver disease } \\
\text { (vs no liver disease) }\end{array}$ & $32(17.2)$ & $4(4.4)$ & .03 & $1.26(1.02-1.56)$ \\
\hline Veterans with cancer and a mental health diagnosis ${ }^{a}$ & $15(8.1)$ & $0(0)$ & .048 & $2.73(0.98-7.58)$ \\
\hline $\begin{array}{l}\text { Veterans with cancer and liver disease who served in Vietnam } \\
\text { (vs other conflicts) }\end{array}$ & $22(11.8)$ & $3(3.3)$ & .04 & $1.40(1.02-2.0)$ \\
\hline Cancer, liver disease, and alcohol and/or drug abuse & $18(9.7)$ & $1(1.1)$ & .01 & $1.43(1.07-1.91)$ \\
\hline
\end{tabular}

aBipolar disorder, schizophrenia, or posttraumatic stress disorder.

and anticholinergic medications $(P=.01 ; \mathrm{OR}$, 2.06; $95 \%$ CI, 1.21-3.52) also increased the risk of delirium (Table 2).

When risk factors were combined, interaction terms were identified (Table 3 ). Those patients found to be at a higher risk of terminal delirium included Vietnam-era veterans with liver disease $(P=.04$; OR, 1.21; $95 \%$ CI, 1.01-1.45) and veterans with a history of drug or alcohol abuse plus comorbid liver disease $(P=.03$; OR, 1.26 ; $95 \% \mathrm{CI}$, $1.02-1.56)$. In a stratified analysis in veterans with a terminal diagnosis of cancer, those with a mental health condition (eg, PTSD, bipolar disorder, or schizophrenia) $(P=.048$; OR, 2.73; 95\% CI, 0.98-7.58) also had higher risk of delirium, though not statistically significant. Within the cancer cohort, veterans with liver disease and a history of drug/alcohol abuse had increased risk of delirium ( $P=.01 ;$ OR, 1.43 ; 95\% CI, 1.07-1.91).

\section{DISCUSSION}

Terminal delirium is experienced by many individuals in their last days to weeks of life. Symptoms can present as hyperactive (eg, agitation, hallucinations, heightened arousal) or hypoactive (lethargy, reduced motor activity, incoherent speech). Hyperactive terminal delirium is particularly problematic because it causes increased distress to the patient, family, and caregivers. Delirium can lead to safety concerns, such as fall risk, due to patients' decreased insight into functional decline.

Many studies suggest both nonpharmacologic and pharmacologic treatments for nonterminal delirium that may also apply to terminal delirium. Nonpharmacologic methods, such as providing a quiet and familiar environment, relieving urinary retention or constipation, and attending to sensory deficits may help prevent or minimize delirium. Pharmacologic interventions, such as antipsychotics or benzodiazepines, may benefit when other modalities have failed to assuage distressing symptoms of delirium. Because hypoactive delirium is usually accompanied by somnolence and reduced motor activity, medication is most often administered to individuals with hyperactive delirium.

The VA provides long-term care hospice beds in their CLCs for veterans who are nearing end of life and have inadequate caregiver support for comprehensive endof-life care in the home (Case Presentation). Because of their military service and other factors common in their life histories, they may have a unique set of characteristics that are predictive of developing terminal delirium. Awareness of the propensity for terminal delirium will allow for early identification of symptoms, timely initiation of nonpharmacologic interventions, and potentially a decreased need for use of antipsychotic medications.

In this study, as noted in previous studies, certain medications (eg, steroids, opioids, and anticholinergics) increased the risk of developing terminal delirium in this veteran population. Steroids and opioids are commonly used in management of neoplasm-related pain and are prescribed throughout the course of terminal illness. The utility of these medications often outweighs potential adverse effects but should 
be considered when assessing the risk for development of delirium. Anticholinergics (eg, glycopyrrolate or scopolamine) are often prescribed in the last days of life for terminal secretions despite lack of evidence of patient benefit. Nonetheless, anticholinergics are used to reduce family and caregiver distress resulting from bothersome sounds from terminal secretions, referred to as the death rattle. ${ }^{21}$

It was found that veterans in the control group lived longer on the hospice unit. It is unclear whether the severity of illness was related to the development of terminal delirium or whether the development of terminal delirium contributed to a hastened death. Veterans with a suspected infection were identified by the use of antibiotics on admission to the hospice unit or when antibiotics were prescribed during the last 2 weeks of life. Thus, treatment of the underlying infection may have contributed to the finding of less delirium in the control group.

More than half the veterans in this study received at least 1 dose of an antipsychotic in the last 2 weeks of life for the treatment of terminal delirium. The most commonly administered medication was haloperidol, given either orally or subcutaneously. Atypical antipsychotics were used less often and were sometimes transitioned to subcutaneous haloperidol as the ability to swallow declined if symptoms persisted.

In this veteran population, having a history of drug or alcohol abuse (even if not recent) increased the risk of terminal delirium. Comorbid cancer and history of mental health disease (eg, PTSD, schizophrenia, bipolar disorder) and Vietnam-era veterans with liver disease (primary cancer, metastases, or cirrhosis) also were more likely to develop terminal delirium.

Just as hospice care is being provided in community settings, nurses are at the forefront of symptom management for veterans residing in VA CLCs under hospice care. Nonpharmacologic interventions are provided by the around-the-clock bedside team to provide comfort for veterans, families, and caregivers throughout the dying process. Nurses' assessment skills and documentation inform the plan of care for the entire interdisciplinary hospice team. Be- cause the treatment of terminal delirium often involves the administration of antipsychotic medications, scrutiny is applied to documentation surrounding these medications. ${ }^{7}$ This study suggested that there is a need for a more rigorous and consistent method of documenting the assessment of, and interventions for, terminal delirium.

\section{Limitations}

Limitations to the current study include hyperactive delirium that was misinterpreted and treated as pain; the probable underreporting of hypoactive delirium and associated symptoms; the use of antipsychotics as a surrogate marker for the development of terminal delirium; and lack of nursing documentation of assessment and interventions of terminal delirium. In addition, the total milligrams of antipsychotics administered per patient were not collected. Finally, there was the potential that other risk factors were not identified due to low numbers of veterans with certain diagnoses (eg, dementia).

\section{CONCLUSIONS}

Based on the findings in this study, several steps have been implemented to enhance the care of veterans under hospice care in this CLC: (1) Nurses providing direct patient care have been educated on the assessment by use of the mRASS and treatment of terminal delirium; ${ }^{22}$ (2) A hospice delirium note template has been created that details symptoms of terminal delirium, nonpharmacologic interventions, the use of antipsychotic medications if indicated, and the outcome of interventions; (3) Providers (eg, physician, advanced practice nurses) review each veteran's medical history for the risk factors noted above; (4) Any risk factor(s) identified by this study will lead to a nursing order for delirium precautions, which requires completion of the delirium note template by nurses each shift.

The goal for this enhanced process is to identify veterans at risk for terminal delirium, observe changes that may indicate the onset of delirium, and intervene promptly to decrease symptom burden and improve quality of life and safety. Potentially, there will be less requirement for the use of antipsychotic medications to control the more 
severe symptoms of terminal delirium. A future study will evaluate the outcome of this enhanced process for the assessment and treatment of terminal delirium in this veteran population.

\section{Acknowledgment}

We thank Martin J. Gorbien, MD, associate chief of staff of Geriatrics and Extended Care, for his continued support throughout this project.

\section{Author affiliations}

Emily Ellsworth, Kevin Bacigalupo, and Kavita Palla are Clinical Pharmacy Specialists in Geriatrics; Seema Limaye is the Director of Hospice and Palliative Medicine; Margare Walkosz is a Nurse Practitioner in Hospice and Palliative Care; and Sandra Szczecinski is the Nursing Manager of the Hospice and Palliative Care Unit; all at Edward Hines, Jr. Veterans Affairs (VA) Hospital. Katie Suda is a Research Health Scientist, Associate Director of Clinical Therapeutics, Center for Health Equity Research and Promotion at VA Pittsburgh Healthcare System and Professor of Medicine at University of Pittsburgh Division of General Internal Medicine both in Pennsylvania.

\section{Author disclosures}

The authors report no actual or potential conflicts of interest with regard to this article.

\section{Disclaimer}

The opinions expressed herein are those of the authors and do not necessarily reflect those of Federal Practitioner, Frontline Medical Communications Inc., the US Government, or any of its agencies. This article may discuss unlabeled or investigational use of certain drugs. Please review the complete prescribing information for specific drugs or drug combinations-including indications, contraindications, warnings, and adverse effects-before administering pharmacologic therapy to patients.

\section{References}

1. Casarett DJ, Inouye SK. Diagnosis and management of delirium near the end of life. Ann Intern Med. 2001;135(1):32-40.

2. American Psychiatric Association. Diagnostic and Statistical Manual of Mental Disorders. 5th ed. Washington, DC; 2013.

3. Grassi L, Caraceni A, Mitchell A, et al. Management of delirium in palliative care: a review. Curr Psychiatry Rep. 2015;17(13):1-9. doi:10.1007/s11920-015-0550-8

4. Bush S, Leonard M, Agar M, et al. End-of-life delirium: issues regarding the recognition, optimal management, and role of sedation in the dying phase. $J$ Pain Symptom Manage. 2014;48 (2):215-230. doi:10.1016/j.jpainsymman. 2014.05.009

5. Moyer D. Terminal delirium in geriatric patients with cancer at end of life. Am J Hosp Palliat Med. 2010;28(1):44-51. doi:10.1177/1049909110376755

6. Lai X, Huang Z, Chen C, et al. Delirium screening in patients in a palliative care ward: a best practice implementation project. JBI Database System Rev Implement Rep.
2019;17(3):429-441. doi:10.11124/JBISRIR-2017-003646

7. Centers for Medicare and Medicaid Services. Medicare and Medicaid Programs; reform of requirements for long-term care facilities. Final rule. Fed Regist. 2016;81 (192):6868868872. Accessed April 17, 2021. https://pubmed.ncbi.nlm nih.gov/27731960

8. Wright $D$, Brajtman $S$, Macdonald M. A relational ethical approach to end-of-life delirium. J Pain Symptom Manage. 2014;48(2):191-198. doi:10.1016/j.jpainsymman.2013.08.015

9. Brajtman S, Higuchi K, McPherson C. Caring for patients with terminal delirium: palliative care unit and home care nurses' experience. Int J Palliat Nurs. 2006;12(4):150-156. doi:10.12968/ijpn.2006.12.4.21010

10. Lange E, Verhaak P, Meer K. Prevalence, presentation, and prognosis of delirium in older people in the population, at home and in long-term care: a review. Int J Geriatr Psychiatry. 2013;28(2):127-134. doi:10.1002/gps.3814

11. Goy E, Ganzini L. Prevalence and natural history of neuropsychiatric syndromes in veteran hospice patients. J Pain Symptom Manage. 2011;41(12):394-401. doi:10.1016/j.jpainsymman.2010.04.015

12. Bush S, Bruera E. The assessment and management of delirium in cancer patients. Oncologist. 2009;4(10):1039-1049. doi:10.1634/theoncologist.2009-0122

13. Clary P, Lawson P. Pharmacologic pearls for end-of-life care. Am Fam Physician. 2009;79(12):1059-1065.

14. Blinderman CD, Billings J. Comfort for patients dying in the hospital. N Engl J Med. 2015;373(26):2549-2561. doi:10.1056/NEJMra1411746

15. Irwin SA, Pirrello RD, Hirst JM, Buckholz GT, Ferris F.D. Clarifying delirium management: practical evidence-based, expert recommendation for clinical practice. J Palliat Med. 2013;16(4):423-435. doi:10.1089/jpm.2012.0319

16. Bobb B. Dyspnea and delirium at the end of life. Clin J Oncol Nurs. 2016;20(3):244-246. doi:10.1188/16.CJON.244-246

17. Morita $\mathrm{T}$, Tei $\mathrm{Y}$, Inoue $\mathrm{S}$. Agitated terminal delirium and association with partial opioid substitution and hydration. J Palliat Med. 2003;6(4):557-563. doi:10.1089/109662103768253669

18. Attard A, Ranjith G, Taylor D. Delirium and its treatment. CNS Drugs. 2008;22(8):631-644-649. doi:10.2165/00023210-200822080-00002

19. Hui D. Benzodiazepines for agitation in patients with delirium: selecting the right patient, right time, and right indication. Curr Opin Support Palliat Care. 2018;12(4):489-494. doi:10.1097/SPC.0000000000000395

20. Irwin P, Murray S, Bilinski A, Chern B, Stafford B. Alcohol withdrawal as an underrated cause of agitated delirium and terminal restlessness in patients with advanced malignancy. J Pain Symptom Manage. 2005;29(1):104-108. doi:10.1016/j.jpainsymman.2004.04.010

21. Lokker ME, van Zuylen $L$, van der Rijt CCD, van der Heide A. Prevalence, impact, and treatment of death rattle: a systematic review. J Pain Symptom Manage. 2014;48:2-12. doi:10.1016/j.jpainsymman.2013.03.011

22. Sessler C, Gosnell M, Grap M, et al. The Richmond Agitation-Sedation Scale: validity and reliability in adult intensive care unit patients. Am J Respir Crit Care Med. 2002:166(10):1338-1344. doi:10.1164/rccm.2107138 